\title{
The UNITE Collaborative: Early Experiences of Introducing Collaborative Trainee Research to Interventional Radiology in the United Kingdom
}

\author{
Indrajeet Mandal $^{1,2}$ (1) $\cdot$ Jim Zhong ${ }^{1,3} \cdot{\text { Robin } \text { Borchert }^{1,4} \cdot \text { Shivank Keni }}^{1,5} \cdot$ \\ Paul Jenkins ${ }^{1,6} \cdot$ Andrew MacCormick ${ }^{1,6}$ - Gregory C. Makris ${ }^{1,7}$
}

Received: 3 July 2021/ Accepted: 25 September 2021/Published online: 19 October 2021

(C) Springer Science+Business Media, LLC, part of Springer Nature and the Cardiovascular and Interventional Radiological Society of Europe (CIRSE) 2021

\section{To the Editor}

The development of research skills has long been an important component in the process of medical and surgical specialities' training, including interventional radiology (IR). However, IR trainees may have fewer opportunities to develop their research acumen [1]. Characteristic of the situation is the fact that according to a recent international IR trainee survey, less than $20 \%$ of IR trainees had completed a PhD/MSc [1] when the equivalent among UK surgical trainees was as high as 50\% [2]. Trainee-led research collaboratives are a successful model for producing high-quality, multi-centre research initiatives, while benefiting trainees in terms of gaining research experience and co-authorship on publications.

Indrajeet Mandal

indrajeet.mandal@ouh.nhs.uk

1 UK National Interventional Radiology Trainee Research (UNITE) Collaborative, 63 Lincoln Inn Fields, London WC2A 3JW, UK

2 John Radcliffe Hospital, Oxford University Hospitals NHS Trust, Headley Way, Oxford, Oxfordshire OX3 9DU, UK

3 St James's University Hospital, Leeds Teaching Hospitals NHS Trust, Beckett St, Harehills, Leeds LS9 7TF, UK

4 Department of Clinical Neurosciences, University of Cambridge, Cambridge CB2 0QQ, UK

5 Edinburgh Medical School, University of Edinburgh, 47 Little France Cres, Edinburgh EH16 4TJ, Scotland

6 Peninsula Radiology Academy, University Hospitals Plymouth NHS Trust, Plymouth, Devon PL6 8DH, UK

7 St Thomas' Hospital, Guys and St Thomas NHS Foundation Trust, Westminster Bridge Rd, London SE1 7EH, UK
Other specialties have initiated their own successful trainee research collaboratives including the British Neurosurgical Trainee Research Collaborative (BNTRC) [3] and the Vascular and Endovascular Research Network (VERN) [4]. As IR expands and moves towards becoming an independent specialty, the authors believe that establishing a research network is a crucial step in ensuring the future of our IR workforce is sufficiently trained in research methodology.

The UK National Interventional Radiology Trainee Research (UNITE) Collaborative was formed in January 2021. The aims were to increase interest in academic IR and undertake multi-centre research projects (Fig. 1). The founding committee consisted of members from the British Society of Interventional Radiology Trainee Committee (BSIRT) and Interventional Radiology Juniors (IRJ) groups (www.irjuniors.com) across multiple stages of training. Guidance was provided by consultant-level IR clinicians as well as the British Society of Interventional Radiology (BSIR) Registries \& Audit Committee.

Project proposals were submitted from members of both the BSIRT and IRJ groups. These were reviewed by the UNITE Collaborative steering committee consisting of academically inclined trainees, and an advisory board consisting of interventional radiologists with academic interests. The inaugural project involved a systematic review of the impact of COVID-19 on IR services across the world. The project involved members from multiple stages of training and resulted in a publication [5]. The first multi-centre project selected was the Multicentre Audit of Cholecystostomy and Further intervention (MACAFI) Audit; a retrospective audit investigating the outcomes of percutaneous cholecystostomy for acute cholecystitis with approximately 1100 patients recruited from 36 centres. 


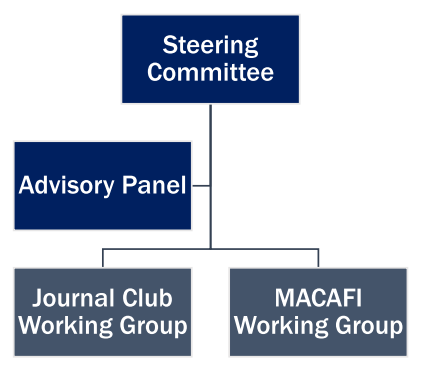

Fig. 1 Organisational structure of the UNITE Collaborative. Each project within the collaborative has a specific working group that is responsible for delivery of the project. All projects are overseen by the steering committee with input from the advisory panel. "MACAFI (Multicentre Audit of Cholecystostomyand Further intervention)"

Further project proposals are reviewed on an ongoing basis, with two further multi-centre studies currently in development.

In addition to research initiatives, this collaborative has placed an emphasis on raising awareness of existing IR research, and a journal club was launched. High impact papers in the field were selected and each session consisted of a trainee presentation of the paper, followed by a multidisciplinary panel discussion in collaboration with other international societies. A total of 8 virtual sessions have been run to date with recordings publicly available following the event. Feedback was obtained from a total of 107 attendees out of a possible 430 - response rate (RR) $25 \%$. A total of $93 \%(\mathrm{n}=100)$ agreed or strongly agreed that the sessions were a good way to keep up with the latest evidence; $80 \%(n=86)$ agreed or strongly agreed that they were more likely to consider a career in academic IR following the sessions. Finally, a dedicated social media account was established to promote the organisation's activities with an emphasis on raising awareness of the collaborative and promoting IR research.

The UNITE collaborative is the first national trainee research network in interventional radiology. The collaborative has shown early promise in conducting multi-centre research projects, running educational initiatives, engaging juniors in academia, and training them to become successful researchers in IR. We hope that this initiative will inspire other junior societies across Europe to establish similar functions and jointly work with us for the benefit of our specialty.

Funding No funding was received to assist with the preparation of this manuscript. The authors have no relevant financial or non-financial interests to disclose.

Human and Animal Rights This article does not contain any studies with human participants or animals performed by any of the authors.

Informed Consent For this type of study, informed consent is not required.

Consent for Publication For this type of study, consent for publication is not required.

\section{References}

1. Makris GC, Burrows V, Lyall F, Moore A, Hamady MS. Vascular and Interventional radiology training; international perspectives and challenges. Cardiovasc Intervent Radiol [Internet]. 2021;44(3):462-72.

2. O'Callaghan J, Mohan HM, Sharrock A, Gokani V, Fitzgerald JE, Williams AP, et al. Cross-sectional study of the financial cost of training to the surgical trainee in the UK and Ireland. BMJ Open. 2017;7(11):e018086.

3. Chari A, Jamjoom AA, Edlmann E, Ahmed AI, Coulter IC, Ma R, et al. The British Neurosurgical trainee research collaborative: five years on. Acta Neurochir (Wien). 2018;160(1):23-8.

4. Bosanquet DC, Stather P, Sidloff DA, Dattani N, Shalhoub J, Pancholi $\mathrm{J}$, et al. How to engage in trainee-led multicentre collaborative vascular research: the vascular and endovascular research network (VERN). Eur J Vasc Endovasc Surg [Internet]. 2016;52(3):392.

5. Xu Y, Mandal I, Lam S, Troumpoukis N, Uberoi R, Sabharwal T, et al. Impact of the COVID-19 pandemic on interventional radiology services across the world. Clin Radiol [Internet]. 2021;76(8):621-5.

Publisher's Note Springer Nature remains neutral with regard to jurisdictional claims in published maps and institutional affiliations. 